\title{
The importance of real-world data to precision medicine
}

\author{
Dipak Kalra*,1 \\ ${ }^{1}$ Department of Medical Informatics and Statistics, University of Gent, Gent 9000, Belgium \\ *Author for correspondence: dipak-kalra@i-hd.eu
}

\section{"Access to fine-grained health data is the next scalability challenge for personalized medicine."}

First draft submitted: 8 October 2018; Accepted for publication: 14 December 2018; Published online: 6 February 2019

Keywords: big data $\bullet$ common data model • data protection • federated data analysis $\bullet$ real world data

Personalizing medical treatment and healthcare delivery on the basis of a person's biology is not new. Administering blood transfusions and differentiating the treatment of sickle-cell disease from carrier status are very old examples. However, what is accelerating the momentum toward more personalized medicine is the deep characterization of individuals by genome, proteome, metabolome or microbiome allowing very precise specificity, alongside the development of (usually) genetically targeted therapies that allows this precise specification to deliver more effective and safer treatments [1]. This widely heralded opportunity has triggered substantial governmental and industry investments, for example in the USA $[2,3]$ and a new European Union Declaration of Co-operation toward access to at least 1 million sequenced genomes in the EU by 2022 [4]. These commitments and investments will accelerate the research that helps to identify how new generation therapies can be more rapidly identified for relevant molecular targets.

Cancers [5] and rare diseases [6] are widely perceived to be the early wins, primarily because therapies can be easily directed toward manifestations of a single gene or sequence with limited requirement for collateral clinical data and minimal dependence on lifestyle or wellness data. These first-line conditions are important areas for societal benefit and are to be welcomed. However, the promise of personalized medicine is greater than this, and making inroads into some of society's greatest healthcare burdens, such as long-term conditions and their primary/secondary prevention, requires much richer clinical and patient-generated data alongside the molecular data, in order to meaningfully characterize patients into subgroups for whom treatments, care pathways and prevention strategies can be better tailored. Artificial intelligence informed care decisions are another form of personalization that is receiving attention and investment, but is not often referred to as a part of personalized medicine - which it is. A wider range of health and care information is needed for machine learning, probably requiring the complete electronic health record (EHR) and increasingly requiring patient-generated monitoring and lifestyle data.

Access to fine-grained health data is the next scalability challenge for personalized medicine. New evidence derived from large populations of data is needed to direct the development of new targeted drugs, to discover unmet needs and the value of novel therapies. An evidence-based reorganization of healthcare is also needed to deliver more personalized packages of care.

Infrastructures providing large populations of detailed health records, alongside genotypic information and/or bio-samples, are a critical success factor to scaling up personalized medicine [7]. Biobanks, as a high-fidelity resource of health data and samples, are not new (e.g., the Estonian BioBank was started in 1999 [8]). What is new and challenging is how to conduct research on large networks of routinely collected health data (real-world data) on the broad range of patients who reflect the true diversity of persons with a condition and not only those who volunteer for clinical trials or biobanks. Some countries have established multiple patient registries that collect and clean routine EHR data for use in healthcare quality improvement and in research, perhaps in Europe the most well-known being Sweden. However, single-disease registries, despite their value, are also no longer sufficient if we are to address the next European and global challenge of multi-morbidity. In recent years we have seen national 
investments in richer eResearch networks, such as the Farr Institute [9] (now Health Data Research UK [10]) and the Medical Informatics Initiative Germany [11], offering comprehensive population coverage.

At a European level the European Medical Informatics Framework (EMIF) has just concluded 5.5 years of research to develop an infrastructure, platform and tools to scale up federated access to EHRs and longitudinal cohort study data [12]. EMIF was sponsored through the EU's Innovative Medicines Initiative [13], a public private research and development partnership between the European Commission and the European Federation of Pharmaceutical Industries and Associations [14]. The EMIF consortium comprised of 58 industry and academic partners with a budget of almost 60 million Euro (approximately half in cash to academic partners and match funded by in kind industry contributions).

The EMIF platform provides an efficient integrated framework for the large-scale re-use of health and life sciences data. The platform enables research users and data sources to collaborate throughout the research lifecycle from data discovery to data sharing and data analysis. It enables approved users to securely analyze multiple, diverse data via a single portal, thereby facilitating research opportunities across a large quantity of research data. The EMIF project included two specific research topics that have helped to guide the development of the platform: the identification and validation of protective and precipitating factors for conversion to Alzheimer's disease, and predictors of metabolic complications of obesity. These two clinical research sub-teams have already published results from EMIF-supported big data research $[15,16]$.

EMIF identified several components that are needed for a large scale federated data access infrastructure, which are also likely to be those needed by any other research data federation.

- A catalog of data sources provided as an online discovery service with rich and searchable metadata that would allow a researcher to locate sources those most likely to have relevant subjects, data and access conditions to support the intended research.

- Data suitability: profiling of the data within one or more data sources to verify if the relevant subjects and data exist, with sufficient patient numbers, to underpin the research.

- Participant and variable selection tools to more precisely specify a research data set that may then be requested for in depth analysis.

- A code of practice defining good practices in data sharing and in protecting data subject privacy (including compliance with the EU General Data Protection Regulation).

- A private remote research environment: a virtual safe haven in which one or more permitted data sets can be analyzed as part of an approved study protocol.

An important challenge faced by EMIF, as by all federated research networks, is data harmonization. Given the diversity of modeling and terminology standards used by EHR system vendors and nations (and, even worse, some EHR systems that do not support standards at all), there is an inevitable need today to undertake some data transformation into a single structural and semantic representation to enable the analysis. EMIF made the choice of using the Observational Medical Outcomes Partnership [17] common data model as its harmonization target, and defined a library of 'knowledge objects' to formalize the semantic representation of the concepts of research interest, primarily for Alzheimer's disease [18].

The use of common data models is a necessity for the moment, but our research and healthcare communities need to unite to promote the better adoption of interoperability standards across the health ecosystem, so that all actors can learn from large scale health data to improve care as well as conduct research. New generation standards must take on board the knowledge discovery needs from all of these perspectives, so that we no longer propagate separate suites of standards for research and for healthcare. This is the vision of the recently formed European Institute for Innovation through Health Data (i $\sim \mathrm{HD}$ ), whose ambition is to bring multiple stakeholders together (healthcare, research, public health, patients and industry), to cocreate solutions for the capture and sharing of better quality health data and its trustworthy use for better health care and efficient research [19].

Of paramount importance to establishing a trusted federated research ecosystem is the need for compliance with data protection legislation, at a European level and across all European member states. The General Data Protection Regulation is currently in the spotlight. Although it is supportive of research, there are concerns that there will be different national interpretations about the correct legal basis for conducting research on routinely collected health data, which will make multi-national research challenging. There are also differing opinions on the acceptability of generic wording to describe future intended research when seeking informed subject consent. 
The status of pseudonymized data and implications of the 'right to be forgotten' on longitudinal research are also concerns. There is uncertainty about how the principle of data minimization applies to big data being curated as a long-term resource.

One cornerstone of ensuring regulatory compliance and assuring trustworthiness is the definition and adoption of good practices: for example, codes of practice, and standard operating rules and procedures. Another is the quality labeling of clinical research platforms and tools to ensure the robustness of their privacy protection measures. Staff training and accreditation is also important, in particular the training of clinical research staff in the appropriate handling of data in safe havens and in the conduct of remote queries. These are all essential components of assuring the trustworthiness of large-scale health data reuse. Without these commitments to the public we will not be able to scale up the reuse of comprehensive population health data.

Society as a whole has varied understandings of how clinical research is conducted, and in particular the importance of health data to research such as in precision medicine. It is therefore important to promote the value of research to the public, and how people's data can be used to deliver health benefits while their privacy remains protected. This is vital if we are to win greater societal endorsement of public health and research uses of health data.

Financial \& competing interests disclosure

The author has no relevant affiliations or financial involvement with any organization or entity with a financial interest in or financial conflict with the subject matter or materials discussed in the manuscript. This includes employment, consultancies, honoraria, stock ownership or options, expert testimony, grants or patents received or pending, or royalties. No specific funding was awarded or donated toward this article. The projects described in it were funded through European Commission grants of which the author was a recipient as a project partner.

No writing assistance was utilized in the production of this manuscript.

\section{Open access}

This article is distributed under the terms of the Creative Commons Attribution License 4.0 which permits any use, distribution, and reproduction in any medium, provided the original author(s) and the source are credited. To view a copy of the license, visit http://creativecommons.org/licenses/by/4.0/

\section{References}

1. Schleidgen S, Klingler C, Bertram T, Rogowski W, Marckmann G. What is personalized medicine: sharpening a vague term based on a systematic literature review. BMC Medical Ethics 14, 55 (2013).

2. Collins F, Varmus H. A new initiative on precision medicine. N. Engl. J. Med. 372, 793-795 (2015).

3. Roche. Partnering with patients in drug development (2018). www.roche.com/media/store/roche_stories/roche-stories-2014-10-20.htm

4. European Commission. EU countries will cooperate in linking genomic databases across borders (2018). https://ec.europa.eu/digital-single-market/en/news/eu-countries-will-cooperate-linking-genomic-databases-across-borders

5. Yates LR, Seoane J, Le Tourneau C, Siu LL, Marais R, Michiels S et al. The European Society for Medical Oncology (ESMO) precision medicine glossary. Ann. Oncol. 29(1), 30-35 (2018).

6. Delavan B, Roberts R, Huang R, Bao W, Tong W, Liu Z. Computational drug repositioning for rare diseases in the era of precision medicine. Drug Discovery Today 23, 382-394 (2018).

7. Aronson S, Rehm H. Building the foundation for genomics in precision medicine. Nature 526, 336-342 (2015).

8. Milani L, Leitsalu L, Metspalu A. An epidemiological perspective of personalized medicine: the Estonian experience. J. Intern. Med. 277, 188-200 (2015).

9. The Farr Institute (2018). www.farrinstitute.org

10. Health Data Research UK (2018). www.hdruk.ac.uk

11. Medical Informatics Initiative Germany (2018). www.medizininformatik-initiative.de/en

12. European Medical Informatics Framework (2018). www.emif.eu

13. The Innovative Medicines Initiative (2018). www.imi.europa.eu

14. European Federation of Pharmaceutical Industries and Associations (2018). www.ef pia.eu

15. Vaudano E, Vannieuwenhuyse B, Van Der Geyten S, van der Lei J, Visser PJ, Streffer J et al. Boosting translational research on Alzheimer's disease in Europe: the innovative medicine initiative AD research platform. Alzheimers Dement. 11(9), 1121-1122 (2015).

16. Roberto G, Leal I, Sattar N, Loomis AK, Avillach P, Egger P et al. Identifying cases of type 2 diabetes in heterogeneous data sources: strategy from the EMIF project. PLoS ONE 11(8), 1-18, e0160648, (2016).

17. The OMOP Common Data Model (2018). www.ohdsi.org/data-standardization/the-common-data-model 
18. Cunningham JA, Van Speybroeck M, Kalra D, Verbeeck R. Nine principles of semantic harmonization. AMIA Annu. Symp. Proc. 2016, 451-459 (2016).

19. Kalra D, Stroetmann V, Sundgren M, Dupont D, Schlünder I, Thienpont G et al. The European Institute for innovation through health data. Learning Health Sys. 1(1), 1-8, e10008, (2016). 